\title{
Concurrency-Induced Transitions in Epidemic Dynamics on Temporal Networks
}

\author{
Tomokatsu Onaga, ${ }^{1,2}$ James P. Gleeson, ${ }^{2}$ and Naoki Masuda," \\ ${ }^{1}$ Department of Physics, Kyoto University, Kyoto 606-8502, Japan \\ ${ }^{2}$ MACSI, Department of Mathematics and Statistics, University of Limerick, Limerick V94 T9PX, Ireland \\ ${ }^{3}$ Department of Engineering Mathematics, University of Bristol, Woodland Road, Bristol BS8 1UB, United Kingdom
}

(Received 16 February 2017; revised manuscript received 13 June 2017; published 6 September 2017)

\begin{abstract}
Social contact networks underlying epidemic processes in humans and animals are highly dynamic. The spreading of infections on such temporal networks can differ dramatically from spreading on static networks. We theoretically investigate the effects of concurrency, the number of neighbors that a node has at a given time point, on the epidemic threshold in the stochastic susceptible-infected-susceptible dynamics on temporal network models. We show that network dynamics can suppress epidemics (i.e., yield a higher epidemic threshold) when the node's concurrency is low, but can also enhance epidemics when the concurrency is high. We analytically determine different phases of this concurrency-induced transition, and confirm our results with numerical simulations.
\end{abstract}

DOI: 10.1103/PhysRevLett.119.108301

Introduction.-Social contact networks-on which infectious diseases occur in humans and animals or viral information spreads online and offline-are mostly dynamic. Switching of partners and the (usually nonMarkovian) activity of individuals, for example, shape network dynamics on such temporal networks [1-3]. A better understanding of epidemic dynamics on temporal networks is needed to help improve predictions of, and interventions in, emergent infectious diseases, to design vaccination strategies, and to identify viral marketing opportunities. This is particularly so because what we know about epidemic processes on static networks [4-7] is only valid when the time scales of the network dynamics and of the infectious processes are well separated. In fact, temporal properties of networks, such as long-tailed distributions of intercontact times, temporal and cross-edge correlation in intercontact times, and entries and exits of nodes, considerably alter how infections spread in a network $[1-3,8,9]$.

In the present study, we focus on a relatively neglected component of temporal networks, i.e., the number of concurrent contacts that a node has. Even if two temporal networks are the same when aggregated over a time horizon, they may be different as temporal networks due to different levels of concurrency. Concurrency is a longstanding concept in epidemiology, in particular in the context of monogamy or polygamy affecting sexually transmitted infections [10-12]. Modeling studies to date largely agree that a level of high concurrency (e.g., polygamy as opposed to monogamy) enhances epidemic spreading in a population. However, this finding, while intuitive, lacks theoretical underpinning. First, some models assume that the mean degree, or equivalently the average contact rate, of nodes increases as the concurrency increases [13-16]. In these cases, the observed enhancement in epidemic spreading is an obvious outcome of a higher density of edges rather than a high concurrency. Second, other models that vary the level of concurrency while preserving the mean degree are numerical $[10,11,17,18]$. In the present study, we use the analytically tractable activity-driven model of temporal networks [19-23] to explicitly modulate the size of the concurrently active network with the structure of the aggregate network fixed. With this machinery, we carefully treat extinction effects, derive an analytically tractable matrix equation using a probability generating function for dynamical networks, and reveal nonmonotonic effects of link concurrency on spreading dynamics. We show that the dynamics of networks can either enhance or suppress infection, depending on the amount of concurrency that individual nodes have. Note that analysis of epidemic processes driven by discrete pairwise contact events, which is a popular approach [1-3,9,23-27], does not address the problem of concurrency because we must be able to control the number of simultaneously active links possessed by a node in order to examine the role of concurrency without confounding with other aspects.

Model.-We consider the following continuous-time susceptible-infected-susceptible (SIS) model on a discrete-time variant of activity-driven networks, which is a generative model of temporal networks [19-23]. The number of nodes is denoted by $N$. Each node $i(1 \leq i \leq N)$ is assigned an activity potential $a_{i}$, drawn from a probability density $F(a) \quad(0<a \leq 1)$. Activity potential $a_{i}$ is the probability with which node $i$ is activated in a window of constant duration $\tau$. If activated, node $i$ creates $m$ undirected links each of which connects to a randomly selected node

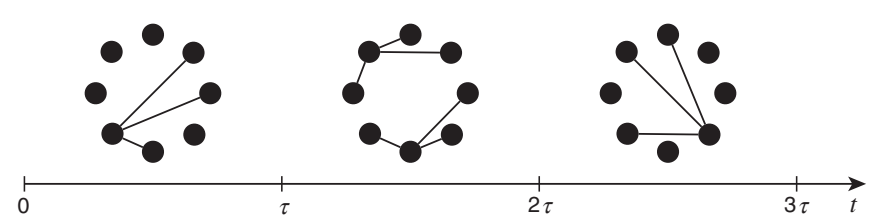

FIG. 1. Schematic of an activity-driven network with $m=3$. 
(Fig. 1). If two nodes are activated and send edges to each other, we only create one edge between them. However, for large $N$ and relatively small $a_{i}$, such events rarely occur. After a fixed time $\tau$, all edges are discarded. Then, in the next time window, each node is again activated with probability $a_{i}$, independently of the activity in the previous time window, and connects to randomly selected nodes by $m$ undirected links. We repeat this procedure. Therefore, the network changes from one time window to another and is an example of a switching network [28-31]. A large $\tau$ implies that network dynamics are slow compared to epidemic dynamics. In the limit of $\tau \rightarrow 0$, the network blinks infinitesimally fast, enabling the dynamical process to be approximated on a time-averaged static network, as in [30].

For the SIS dynamics, each node takes either the susceptible or infected state. At any time, each susceptible node contracts infection at rate $\beta$ per infected neighboring node. Each infected node recovers at rate $\mu$ irrespectively of the neighbors' states. Changing $\tau$ to $c \tau(c>0)$ is equivalent to changing $\beta$ and $\mu$ to $\beta / c$ and $\mu / c$, respectively, while leaving $\tau$ unchanged. Therefore, we set $\mu=1$ without loss of generality.

Analysis.-We calculate the epidemic threshold as follows. First, we formulate SIS dynamics near the epidemic threshold on a static star graph, which is the building block of the activity-driven model, while explicitly considering extinction effects. Second, we convert the obtained set of linear difference equations into a tractable mathematical form with the use of a probability generating function of an activity distribution. Third, the epidemic threshold is obtained from an implicit function. For the sake of the analysis, we assume that star graphs generated by an activated node, which we call the hub, are disjoint from each other. Because a star graph with hub node $i$ overlaps with another star graph with probability $\approx m \sum_{j \neq i} a_{j}(m+1) / N \propto m^{2}\langle a\rangle$, where $\langle a\rangle \equiv$ $\int d a F(a) a$ is the mean activity potential, we impose $m^{2}\langle a\rangle \ll 1$. (However, our method works better than the so-called individual-based approximation even when $m^{2}\langle a\rangle=0.5$, as shown in the Supplemental Material [32].) We denote by $\rho(a, t)$ the probability that a node with activity $a$ is infected at time $t$. The fraction of infected nodes in the entire network at time $t$ is given by $\langle\rho(t)\rangle \equiv \int d a F(a) \rho(a, t)$. Let $c_{1}$ be the probability with which the hub in an isolated star graph is infected at time $t+\tau$, when the hub is the only infected node at time $t$ and the network has switched to a new configuration right at time $t$. Let $c_{2}$ be the probability with which the hub is infected at $t+\tau$ when only a single leaf node is infected at $t$. The probability that a hub with activity potential $a$ is infected after the duration $\tau$ of the star graph, denoted by $\rho_{1}$, is given by

$$
\rho_{1}(a, t+\tau)=c_{1} \rho(a, t)+c_{2} m\langle\rho(t)\rangle .
$$

In deriving Eq. (1), we considered the situation near the epidemic threshold such that at most one node is infected in the star graph at time $t$ [and hence $\rho(a, t),\langle\rho(t)\rangle \ll 1$ ]. The probability that a leaf with activity potential $a$ that has a hub neighbor with activity potential $a^{\prime}$ is infected after time $\tau$ is analogously given by

$$
\begin{aligned}
\rho_{2}\left(a, a^{\prime}, t+\tau\right)= & c_{3} \rho(a, t)+c_{4} \rho\left(a^{\prime}, t\right) \\
& +c_{5}(m-1)\langle\rho(t)\rangle,
\end{aligned}
$$

where $c_{3}, c_{4}$, and $c_{5}$ are the probabilities with which a leaf node with activity potential $a$ is infected after duration $\tau$ when only that leaf node, the hub, and a different leaf node is infected at time $t$, respectively. We derive formulas for $c_{i}$ $(1 \leq i \leq 5)$ in the Supplemental Material [32]. The probability that an isolated node with activity potential $a$ is infected after time $\tau$ is given by $e^{-\tau} \rho(a, t)$. By combining these contributions, we obtain

$$
\begin{aligned}
\rho(a, t+\tau)= & a \rho_{1}(a, t+\tau)+\int d a^{\prime} F\left(a^{\prime}\right) m a^{\prime} \rho_{2}\left(a, a^{\prime}, t+\tau\right) \\
& +(1-a-m\langle a\rangle) e^{-\tau} \rho(a, t) .
\end{aligned}
$$

To analyze Eq. (3) further, we take a generating function approach. With this approach, one trades a probability distribution for a probability generating function whose derivatives provide us with useful information about the distribution such as its moments. Furthermore, it often makes analysis easier, in particular linear analysis. By multiplying Eq. (3) by $z^{a}$ and averaging over $a$, we obtain

$$
\begin{aligned}
\Theta(z, t+\tau)= & c_{1}^{\prime} \Theta^{(1)}(z, t)+c_{2}^{\prime} \Theta(1, t) g^{(1)}(z)+c_{3}^{\prime} \Theta(z, t) \\
& +\left[c_{4}^{\prime} \Theta^{(1)}(1, t)+c_{5}^{\prime} \Theta(1, t)\right] g(z),
\end{aligned}
$$

where $c_{1}^{\prime} \equiv c_{1}-e^{-\tau}, c_{2}^{\prime} \equiv m c_{2}, c_{3}^{\prime} \equiv e^{-\tau}+m\langle a\rangle\left(c_{3}-e^{-\tau}\right)$, $c_{4}^{\prime} \equiv m c_{4}, \quad c_{5}^{\prime} \equiv m(m-1)\langle a\rangle c_{5}, \quad g(z) \equiv \int d a F(a) z^{a}$ is the probability generating function of $a, \Theta(z, t) \equiv$ $\int d a F(a) \rho(a, t) z^{a}$, and throughout the paper the superscript $(n)$ represents the $n$th derivative with respect to $\ln z$. Although Eq. (3) is an infinite dimensional system of linear difference equations, Eq. (4) is a single difference equation of $\Theta(z, t)$ and its derivative [35].

We expand $\rho(a, t)$ as a Maclaurin series as follows:

$$
\rho(a, t)=\sum_{n=1}^{\infty} w_{n}(t) a^{n-1} .
$$

Using this polynomial basis representation (the convergence is proven in the Supplemental Material [32]), we can consider the differentiations in Eq. (4) [i.e., $\Theta^{(1)}(z, t)$ and $\left.g^{(1)}(z)\right]$ as an exchange of bases and convert Eq. (4) into a tractable matrix form. Let $p_{0}$ be the fraction of initially infected nodes, which are selected uniformly at random, independently of $a$. We represent the initial condition as $\boldsymbol{w}(t=0) \equiv\left(w_{1}(0), w_{2}(0), \ldots\right)^{\top}=\left(p_{0}, 0,0, \ldots\right)^{\top}$.

Epidemic dynamics near the epidemic threshold obey linear dynamics given by

$$
\boldsymbol{w}(t+\tau)=\boldsymbol{T}(\tau) \boldsymbol{w}(t) .
$$


By substituting $\Theta(z, t)=\sum_{n=1}^{\infty} w_{n}(t) g^{(n-1)}(z)$ and $g^{(n-1)}(1)=\left\langle a^{n-1}\right\rangle$ in Eq. (4), we obtain

$$
\boldsymbol{T}=\left(\begin{array}{cccccc}
c_{3}^{\prime}+\langle a\rangle c_{4}^{\prime}+c_{5}^{\prime} & \left\langle a^{2}\right\rangle c_{4}^{\prime}+\langle a\rangle c_{5}^{\prime} & \left\langle a^{3}\right\rangle c_{4}^{\prime}+\left\langle a^{2}\right\rangle c_{5}^{\prime} & \left\langle a^{4}\right\rangle c_{4}^{\prime}+\left\langle a^{3}\right\rangle c_{5}^{\prime} & \left\langle a^{5}\right\rangle c_{4}^{\prime}+\left\langle a^{4}\right\rangle c_{5}^{\prime} & \cdots \\
c_{1}^{\prime}+c_{2}^{\prime} & \langle a\rangle c_{2}^{\prime}+c_{3}^{\prime} & \left\langle a^{2}\right\rangle c_{2}^{\prime} & \left\langle a^{3}\right\rangle c_{2}^{\prime} & \left\langle a^{4}\right\rangle c_{2}^{\prime} & \ldots \\
0 & c_{1}^{\prime} & c_{3}^{\prime} & 0 & 0 & \ldots \\
0 & 0 & c_{1}^{\prime} & c_{3}^{\prime} & 0 & \ldots \\
0 & 0 & 0 & c_{1}^{\prime} & c_{3}^{\prime} & \ldots \\
\vdots & \vdots & \vdots & \vdots & \vdots & \ddots
\end{array}\right) .
$$

A positive prevalence $\langle\rho(t)\rangle$ (i.e., a positive fraction of infected nodes in the equilibrium state) occurs only if the largest eigenvalue of $\boldsymbol{T}(\tau)$ exceeds 1 , because in this situation the probability of being infected grows in time, at least in the linear regime. Therefore, we get the following implicit function for the epidemic threshold, denoted by $\beta_{c}$ :

$$
\begin{aligned}
f\left(\tau, \beta_{c}\right) \equiv & \frac{(1-r)(1-s)-(1+q) u}{S(q)} \\
& -q r-q s+q r s-q^{2} u-r s=0,
\end{aligned}
$$

where $S(q) \equiv \sum_{n=0}^{\infty}\left(\left\langle a^{n+2}\right\rangle /\langle a\rangle^{n+2}\right) q^{n}=\left(1 /\langle a\rangle^{2}\right)\left\{\left\langle\left(a^{2}\right) /\right.\right.$ $[1-(a /\langle a\rangle) q]\rangle\}, q \equiv\langle a\rangle c_{1}^{\prime} /\left(1-c_{3}^{\prime}\right), r \equiv\langle a\rangle c_{2}^{\prime} /\left(1-c_{3}^{\prime}\right)$, $s \equiv\langle a\rangle c_{4}^{\prime} /\left(1-c_{3}^{\prime}\right)$, and $u \equiv c_{5}^{\prime} /\left(1-c_{3}^{\prime}\right)$ (see Supplemental Material [32] for the derivation). Note that $f$ is a function of $\beta\left(=\beta_{c}\right)$ through $q, r, s$, and $u$, which are functions of $\beta$. In general, we obtain $\beta_{c}$ by numerically solving Eq. (8), but some special cases can be determined analytically.

In the limit $\tau \rightarrow 0$, Eq. (8) gives $\beta_{c}=[m(\langle a\rangle+$ $\left.\left.\sqrt{\left\langle a^{2}\right\rangle}\right\rangle\right]^{-1}$, which coincides with the epidemic threshold for the activity-driven model derived in the previous studies $[19,22]$. In fact, this $\beta_{c}$ value is the epidemic threshold for the aggregate (and hence static) network, whose adjacency matrix is given by $A_{i j}^{*} \approx m\left(a_{i}+a_{j}\right) / N[3,31]$, as demonstrated in Fig. S1 [32].

For general $\tau$, if all nodes have the same activity potential $a$, and if $m=1$, we obtain $\beta_{c}$ as the solution of the following implicit equation:

$$
\begin{gathered}
2 a e^{\left[\left(\beta_{c}-1\right) \tau / 2\right]}\left[\cosh \left(\frac{\kappa_{c} \tau}{2}\right)+\frac{1+3 \beta_{c}}{\kappa_{c}} \sinh \left(\frac{-\kappa_{c} \tau}{2}\right)\right] \\
-e^{\tau}+1-2 a=0,
\end{gathered}
$$

where $\kappa_{c}=\sqrt{\beta_{c}^{2}+6 \beta_{c}+1}$.

The theoretical estimate of the epidemic threshold [Eq. (8); we use Eq. (9) in the case of $m=1$ ] is shown by the solid lines in Figs. 2(a) and 2(b). It is compared with numerically calculated prevalence values for various $\tau$ and $\beta$ values shown in different colors. Equations (8) and (9) describe the numerical results fairly well. When $m=1$, the epidemic threshold increases with $\tau$ and diverges at $\tau \approx 0.1$ [Fig. 2(a)]. Furthermore, slower network dynamics (i.e., larger values of $\tau$ ) reduce the prevalence for all values of $\beta$. In contrast, when $m=10$, the epidemic threshold decreases and then increases as $\tau$ increases [Fig. 2(b)]. The network dynamics (i.e., finite $\tau$ ) impact epidemic dynamics in a qualitatively different manner depending on $m$, i.e., the number of concurrent neighbors that a hub has. Note that the estimate of $\beta_{c}$ by the individual-based approximation ([31], see Supplemental Material [32] for the derivation), which may be justified when $m \gg 1$, is consistent with the numerical results and our theoretical results only at small $\tau$ [a dashed line in Fig. 2(b)].
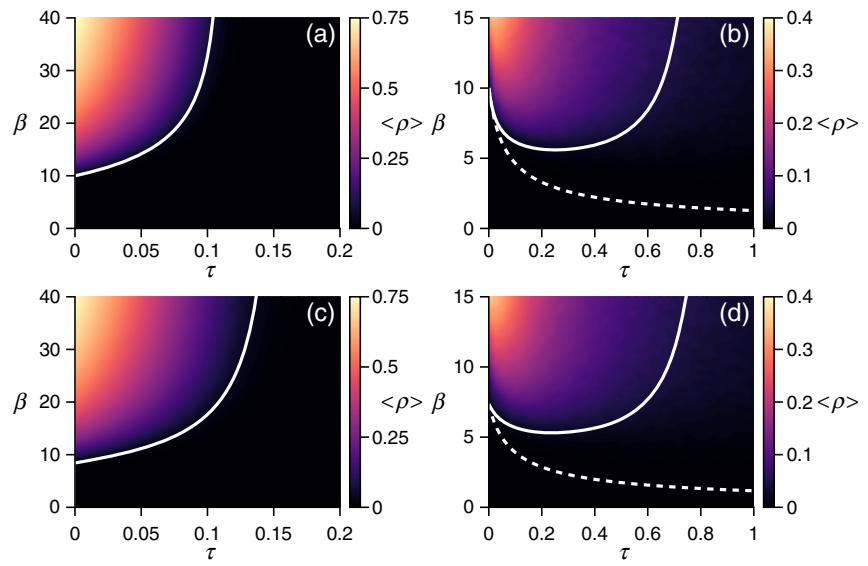

FIG. 2. Epidemic threshold and the numerically-simulated prevalence when $m=1$ (a),(c) and $m=10$ (b),(d). In (a) and (b), all nodes have the same activity potential value $a$. The solid lines represent the analytical estimate of the epidemic threshold [Eq. (8); we plot Eq. (9) instead in (a)]. The dashed lines represent the epidemic threshold obtained from the individual-based approximation (Supplemental Material [32]). The color indicates the prevalence. In (c) and (d), the activity potential $\left(\epsilon \leq a_{i} \leq 0.9,1 \leq i \leq N\right)$ obeys a power-law distribution with exponent 3 . In (a)-(d), we set $N=2000$ and adjust the values of $a$ and $\epsilon$ such that the mean degree is the same $(\langle k\rangle=0.1)$ in the four cases. We simulate the stochastic SIS dynamics using the quasistationary state method [36], as in [31], and calculate the prevalence averaged over 100 realizations after discarding the first 15000 time steps. We set the step size $\Delta t=0.002$. Qualitatively similar results are obtained for the variant of the activity-driven model with a reinforcement mechanism of link creation [37] (Fig. S3 [32]). 

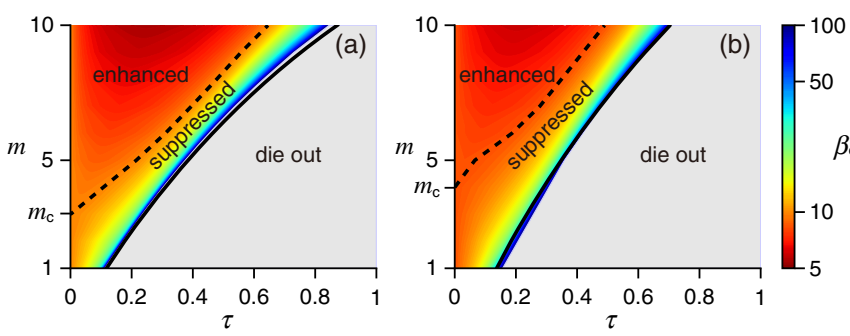

FIG. 3. Phase diagrams for the epidemic threshold, $\beta_{c}$, when the activity potential is (a) equal to $a$ for all nodes, or (b) obeys a power-law distribution with exponent $3\left(\epsilon \leq a_{i} \leq 0.9\right)$. We set $\langle k\rangle=0.1$ at $m=1$ and adjust the value of $a$ and $\epsilon$ such that $\beta_{c}$ takes the same value for all $m$ at $\tau=0$. In the "die out" phase, infection eventually dies out for any finite $\beta$. In the "suppressed" phase, $\beta_{c}$ is larger than the $\beta_{c}$ value at $\tau=0$. In the "enhanced" phase, $\beta_{c}$ is smaller than the $\beta_{c}$ value at $\tau=0$. The solid and dashed lines represent $\tau_{*}$ [Eq. (10)] and $\tau_{c}$, respectively. The color bar indicates the $\beta_{c}$ values. In the gray regions, $\beta_{c}>100$.

Qualitatively similar results are found, when the activity potential $a$ is power-law distributed [Figs. 2(c) and 2(d)].

To illuminate the qualitatively different behaviors of the epidemic threshold as $\tau$ increases, we determine a phase diagram for the epidemic threshold. We focus our analysis on the case in which all nodes share the activity potential value $a$, noting that qualitatively similar results are also found for power-law distributed activity potentials [Fig. 3(b)]. We calculate the two boundaries partitioning different phases as follows. First, we observe that the epidemic threshold diverges at $\tau=\tau_{*}$. In the limit $\beta \rightarrow \infty$, infection starting from a single infected node in a star graph immediately spreads to the entire star graph, leading to $c_{i} \rightarrow 1(1 \leq i \leq 5)$. By substituting $c_{i} \rightarrow 1$ in Eq. (8), we obtain $f\left(\tau_{*}, \beta_{c} \rightarrow \infty\right)=0$, where

$$
\tau_{*}=\ln \frac{1-(1+m) a}{1-(1+m)^{2} a} .
$$

When $\tau>\tau_{*}$, infection always dies out even if the infection rate is infinitely large. This is because, in a finite network, infection always dies out after a sufficiently long time due to stochasticity [38-40]. Second, although $\beta_{c}$ eventually diverges as $\tau$ increases, there may exist $\tau_{c}$ such that $\beta_{c}$ at $\tau<\tau_{c}$ is smaller than the $\beta_{c}$ value at $\tau=0$. Motivated by the comparison between the behavior of $\beta_{c}$ at $m=1$ and $m=10$ (Fig. 2), we postulate that $\tau_{c}(>0)$ exists only for $m>m_{c}$. Then, we obtain $d \beta_{c} / d \tau=0$ at $(\tau, m)=\left(0, m_{c}\right)$. The derivative of Eq. (8) gives $\partial f / \partial \tau+$ $\left(\partial f / \partial \beta_{c}\right)\left(d \beta_{c} / d \tau\right)=0$. Because $d \beta_{c} / d \tau=0$ at $(\tau, m)=$ $\left(0, m_{c}\right)$, we obtain $\partial f / \partial \tau=0$, which leads to

$$
m_{c}=\frac{3}{1-4 a} \text {. }
$$

When $m<m_{c}$, network dynamics (i.e., finite $\tau$ ) always reduce the prevalence for any $\tau$ [Figs. 2(a) and 2(c)]. When $m>m_{c}$, a small $\tau$ raises the prevalence as compared to $\tau=0$ (i.e., static network) but a larger $\tau$ reduces the prevalence [Figs. 2(b) and 2(d)].

The phase diagram based on Eqs. (10) and (11) is shown in Fig. 3(a). The $\beta_{c}$ values numerically calculated by solving Eq. (8) are also shown in the figure. It should be noted that the parameter values are normalized such that $\beta_{c}$ has the same value for all $m$ at $\tau=0$. We find that the dynamics of the network may either increase or decrease the prevalence, depending on the number of connections that a node can simultaneously have, extending the results shown in Fig. 2.

These results are not specific to the activity-driven model. The phase diagram is qualitatively similar for randomly distributed $m$ (Fig. S4 [32]), for different distributions of activity potentials (Fig. S5 [32]), and for a different model in which an activated node induces a clique instead of a star (Fig. S6 [32]), modeling a group conversation event as some temporal network models do [41-43].

Discussion.-Our analytical method shows that the presence of network dynamics boosts the prevalence (and decreases the epidemic threshold $\beta_{c}$ ) when the concurrency $m$ is large and suppresses the prevalence (and increases $\beta_{c}$ ) when $m$ is small, for a range of values of the network dynamic time scale $\tau$. This result lends theoretical support to previous claims that concurrency boosts epidemic spreading [10,11,13-19,44]. The result may sound unsurprising because a large $m$ value implies that there exists a large connected component at any given time. However, our finding is not trivial because a large component consumes many edges such that other parts of the network at the same time or the network at other times would be more sparsely connected as compared to the case of a small $\mathrm{m}$. We confirmed that qualitatively similar results are found when the activity potentials were constructed from two empirical social contact networks (Fig. S7 [32]). Our results confirm that a monogamous sexual relationship or a small group of people chatting face to face, as opposed to polygamous relationships or large groups of conversations, hinders epidemic spreading, where we compare like with like by constraining the aggregate (static) network to be the same in all cases. For general temporal networks, immunization strategies that decrease concurrency (e.g., discouraging polygamy) may be efficient. Restricting the size of the concurrent connected component (e.g., size of a conversation group) may also be a practical strategy.

Another important contribution of the present study is the observation that infection dies out for a sufficiently large $\tau$, regardless of the level of concurrency. As shown in Figs. 3 and S6 [32], the transition to the "die out" phase occurs at values of $\tau$ that correspond to network dynamics and epidemic dynamics having comparable time scales. This is a stochastic effect and cannot be captured by existing approaches to epidemic processes on temporal networks that neglect stochastic dying out, such as differential equation systems for pair formulation-dissolution models [11,15-18] and individual-based approximations $[31,45,46]$. Our analysis methods explicitly consider such 
stochastic effects, and are therefore expected to be useful beyond the activity-driven model (or the clique-based temporal networks analyzed in the Supplemental Material [32]) and the SIS model.

We thank Leo Speidel for discussion. We thank the SocioPatterns collaboration (http:// www.sociopatterns.org) for providing the data set. T. O. acknowledges the support provided through JSPS Research Fellowship for Young Scientists. J. G. acknowledges the support provided through Science Foundation Ireland (Grants No. 15/SPP/ E3125 and No. 11/PI/1026). N. M. acknowledges the support provided through JST, CREST, and JST, ERATO, Kawarabayashi Large Graph Project.

*naoki.masuda@bristol.ac.uk

[1] P. Holme and J. Saramäki, Phys. Rep. 519, 97 (2012).

[2] P. Holme, Eur. Phys. J. B 88, 234 (2015).

[3] N. Masuda and R. Lambiotte, A Guide to Temporal Networks (World Scientific, Singapore, 2016).

[4] M. J. Keeling and K. T. D. Eames, J. R. Soc. Interface 2, 295 (2005).

[5] A. Barrat, M. Barthélemy, and A. Vespignani, Dynamical Processes on Complex Networks (Cambridge University Press, Cambridge, England, 2008).

[6] R. Pastor-Satorras, C. Castellano, P. Van Mieghem, and A. Vespignani, Rev. Mod. Phys. 87, 925 (2015).

[7] M. A. Porter and J. P. Gleeson, Dynamical Systems on Networks, Frontiers in Applied Dynamical Systems: Reviews and Tutorials (Springer International Publishing, Cham, Switzerland, 2016), Vol. 4.

[8] S. Bansal, J. Read, B. Pourbohloul, and L. A. Meyers, J. Biol. Dyn. 4, 478 (2010).

[9] N. Masuda and P. Holme, F1000Prime Rep. 5, 6 (2013).

[10] M. Morris and M. Kretzschmar, Soc. Networks 17, 299 (1995).

[11] M. Kretzschmar and M. Morris, Math. Biosci. 133, 165 (1996).

[12] J. C. Miller and A. C. Slim, arXiv:1611.04800.

[13] C. H. Watts and R. M. May, Math. Biosci. 108, 89 (1992).

[14] I. A. Doherty, S. Shiboski, J. M. Ellen, A. A. Adimora, and N. S. Padian, Sex. Transm. Dis. 33, 368 (2006).

[15] K. Gurski and K. Hoffman, Math. Biosci. 282, 91 (2016).

[16] K. T. D. Eames and M. J. Keeling, Math. Biosci. 189, 115 (2004).

[17] C. Bauch and D. A. Rand, Proc. R. Soc. B 267, 2019 (2000).

[18] K. Y. Leung and M. Kretzschmar, AIDS 29, 1097 (2015).

[19] N. Perra, B. Gonçalves, R. Pastor-Satorras, and A. Vespignani, Sci. Rep. 2, 469 (2012).

[20] M. Starnini and R. Pastor-Satorras, Phys. Rev. E 87, 062807 (2013).

[21] B. Ribeiro, N. Perra, and A. Baronchelli, Sci. Rep. 3, 3006 (2013).

[22] S. Liu, N. Perra, M. Karsai, and A. Vespignani, Phys. Rev. Lett. 112, 118702 (2014).
[23] L. Zino, A. Rizzo, and M. Porfiri, Phys. Rev. Lett. 117, 228302 (2016).

[24] A. Vazquez, B. Rácz, A. Lukács, and A. L. Barabási, Phys. Rev. Lett. 98, 158702 (2007).

[25] M. Karsai, M. Kivelä, R. K. Pan, K. Kaski, J. Kertész, A. L. Barabási, and J. Saramäki, Phys. Rev. E 83, 025102(R) (2011).

[26] G. Miritello, E. Moro, and R. Lara, Phys. Rev. E 83, 045102 (R) (2011).

[27] J. Stehlé, N. Voirin, A. Barrat, C. Cattuto, V. Colizza, L. Isella, C. Régis, J. F. Pinton, N. Khanafer, W. Van den Broeck, and P. Vanhems, BMC Med. 9, 87 (2011).

[28] D. Liberzon, Switching in Systems and Control, Systems and Control: Foundations and Applications (Birkhäuser, Boston, 2003).

[29] N. Masuda, K. Klemm, and V. M. Eguíluz, Phys. Rev. Lett. 111, 188701 (2013).

[30] M. Hasler, V. Belykh, and I. Belykh, SIAM J. Appl. Dyn. Syst. 12, 1007 (2013).

[31] L. Speidel, K. Klemm, V. M. Eguíluz, and N. Masuda, New J. Phys. 18, 073013 (2016).

[32] See Supplemental Material at http://link.aps.org/ supplemental/10.1103/PhysRevLett.119.108301, which includes Refs. [33,34], for details of mathematical derivations and extensions of the model.

[33] M. Génois, C. L. Vestergaard, J. Fournet, A. Panisson, I. Bonmarin, and A. Barrat, Netw. Sci. 3, 326 (2015).

[34] A. Paranjape, A. R. Benson, and J. Leskovec, in Proceedings of the Tenth ACM International Conference on Web Search and Data Mining, WSDM'17 (ACM, New York, 2017), pp. 601-610.

[35] H. Silk, G. Demirel, M. Homer, and T. Gross, New J. Phys. 16, 093051 (2014).

[36] M. M. de Oliveira and R. Dickman, Phys. Rev. E 71, 016129 (2005).

[37] M. Karsai, N. Perra, and A. Vespignani, Sci. Rep. 4, 4001 (2014).

[38] M. J. Keeling and J. V. Ross, J. R. Soc. Interface 5, 171 (2008).

[39] P. L. Simon, M. Taylor, and I. Z. Kiss, J. Math. Biol. 62, 479 (2011).

[40] J. Hindes and I. B. Schwartz, Phys. Rev. Lett. 117, 028302 (2016).

[41] C. Tantipathananandh, T. Berger-Wolf, and D. Kempe, in Proceedings of the Thirteenth ACM SIGKDD International Conference on Knowledge Discovery and Data Mining (ACM, New York, 2007), pp. 717-726.

[42] J. Stehlé, A. Barrat, and G. Bianconi, Phys. Rev. E 81, 035101(R) (2010).

[43] K. Zhao, M. Karsai, and G. Bianconi, PLoS One 6, e28116 (2011).

[44] J. W. Eaton, T. B. Hallett, and G. P. Garnett, AIDS Behav. 15, 687 (2011).

[45] E. Valdano, L. Ferreri, C. Poletto, and V. Colizza, Phys. Rev. X 5, 021005 (2015).

[46] L. E. C. Rocha and N. Masuda, Sci. Rep. 6, 31456 (2016). 